\title{
Desarrollo y autorrealización personal de los trabajadores mineros de socavón en una empresa minera con una gestión de calidad. Huancayo - Diciembre 2016
}

\section{Development and personal self-realization of mining workers in a mining company with quality management. Huancayo - Diciembre 2016}

\author{
Linda Nuñez Inga ${ }^{1}$ \\ Universidad Nacional del Centro del Perú
}

Recibido: $25-03-17$

Aceptado: $01-06-17$

\section{Resumen}

El artículo Desarrollo y Autorrealización Personal de los Trabajadores Mineros de Socavón en una Empresa Minera con una Gestión de Calidad (Caso Empresa Minera Milpo - Mina Cerro Lindo), basada en la Investigación realizado por el autor, "bienestar psicosocial de los trabajadores del Centro Minero Milpo-Chincha Alta-2015", asume dos dimensiones del bienestar psicosocial del ser humano, y el Propósito de Vida y Crecimiento Personal, en relación con una empresa que implementa una gestión de Calidad, para con los resultados obtenidos demostrar que se da una tendencia dominante negativa en relación a estas dimensiones y que es necesario ahondar con criterios científicos la situación del bienestar psicosocial de los trabajadores mineros, como es el caso del objeto de estudio.

Palabras claves: Bienestar psicosocial, factores de riesgo, desarrollo y autorrealización; mineros de socavón, minería.

\begin{abstract}
The article Development and Personal Self - Realization of the Mining Workers of Socavón in a Mining Company with a Quality Management (Case Mining Milpo - Cerro Lindo Mine), based on the research carried out by the author, the psychosocial well - being of the workers of the Mining Center Milpo-Chincha Alta-2015", assumes two dimensions of the psychosocial well-being of the human being, and the Purpose of Life and Personal Growth, in relation to a company that implements a Quality Management, with the obtained results proving that a tendency is given Dominant negative in relation to these dimensions and that it is necessary to deepen with scientific criteria the situation of the psychosocial welfare of the mining workers, as is the case of the object of study.
\end{abstract}

Keywords: Psychosocial well-being, risk factors, development and self-actualization, miners, mining.

\footnotetext{
1. Universidad Nacional del Centro del Perú. Email: lindatarma@hotmail.com
} 
El presente artículo, Desarrollo y Autorrealización Personal de los Trabajadores Mineros de Socavón en una Empresa Minera con una Gestión de Calidad, es un informe basado en la investigación realizada el año 2015, Bienestar Psicosocial de los Trabajadores del Centro Minero MILPO-Chincha Alta, así expone los resultados de la investigación de uno de los componentes esenciales del bienestar psicosocial, como es el Desarrollo y la Autorrealización personal, de los trabajadores mineros que laboran en socavón. El artículo expone los resultados de la investigación en relación al grado de satisfacción de los trabajadores mineros que laboran en socavón, respecto a su Desarrollo y Autorrealización personal, en un estudio de Caso de la Compañía Minera Milpo S.A.A., en la mina Cerro Lindo, ubicada en el Perú, departamento de Ica, provincia de Chincha, distrito de Chavín.

La investigación se da en el contexto de una economía globalizada, donde las empresas modernas implementan y desarrollan gestiones de Calidad, y los Estados se comprometen a cumplir normas internacionales que establecen en forma prioritaria el derecho a la seguridad y a la salud en el trabajo como un derecho fundamental del ser humano, como lo establece el Pacto Internacional de Derechos Económicos, Sociales y Culturales, el cual con arreglo a la Declaración Universal de Derechos Humanos, considera que no puede realizarse el ideal del ser humano libre, liberado del temor y de la miseria, a menos que se creen las condiciones que permitan a cada persona gozar de sus derechos económicos, sociales y culturales, tanto como de sus derechos civiles y políticos. Este instrumento internacional se establece especialmente el derecho de toda persona a gozar de un trabajo en condiciones equitativas y satisfactorias (Carmen \& Idarmis, 2000)

\section{La problemática psicosocial del trabajador minero}

Se considera en la Investigación que los factores de riesgo psicosocial son "aquellas condiciones que se encuentran presentes en una situación laboral y que están directamente relacionadas con la organización, el contenido del trabajo, y la realización de la tarea". (Kayes, 1995). Estos factores son tomados en cuenta en la problemática de los trabajadores mineros, objeto de la investigación. Teniendo presente que la salud del trabajador es cada vez más un tema de preocupación de las organizaciones del siglo XXI, caracterizándose por una mayor conciencia que se traduce en las leyes y la metodología en torno a la prevención de riesgos laborales. (Jaramillo, 2005).

\section{Antecedente}

El artículo expone uno de los aspectos de una Investigación inédita, en relación a su objeto, los trabajadores mineros de la Compañía Milpo S.A.A. y su bienestar psicosocial. Sin embargo, se debe considerar investigaciones similares como antecedentes, destacando a nivel nacional "El papel de las creencias de seguridad en los resultados de seguridad influyentes en el sector minero en los países de América del sur". Guayaquil: Ecuador (Pacheco, 2015). Aborda a los trabajadores 
mineros en los asentamientos mineros de la sierra sur y norte del Perú, analizando y evaluando el conjunto de los riesgos, psicológicos, en las minas de tajo abierto y de socavón. Este perfil ayuda en la orientación psicológica del comportamiento del minero tanto en el nivel individual, familiar y social, se da mayor relevancia a la familia, relaciones interpersonales, y los derechos de los trabajadores.

\section{Base Teórica, La Teoría de lo Social}

La teoría de lo Social de Julio Durand Lazo, expuesta desde finales de los 70, (Durand, 2001), y que desarrolla hasta nuestros días, como teoría sociológica general, permite la comprensión del Bienestar psicosocial de los trabajadores del centro minero MILPO-chincha alta-2015, como efecto de la complementación social entre la empresa con los trabajadores mineros, expresados como lo social en la mente o psique de los trabajadores, en términos de grados satisfacción en su desarrollo y autorrealización personal en sus labores mineras en el socavón.

La complementación social planteada por La teoría de lo Social, se fundamenta en la determinación de lo social como lo más general, siendo el concepto social la abstracción más simple y general del universo sociológico. En la sociedad todo es social o es producto de lo social. Así, es importante conocer como la teoría de lo Social define a lo social, en los siguientes términos: "Lo social es el proceso por el que un ser vivo se realiza con otro ser vivo. Lo social es el proceso por el que un ser vivo se realza en otro ser vivo. Lo social es el proceso por el que un ser vivo se realiza por otro ser vivo. Lo social es el proceso por el que un ser vivo se realiza para otro ser vivo. Esta definición es una: Lo Social es el proceso porque un ser vivo se realiza CON, POR, EN, PARA, otro ser vivo. Esta definición es extensivo al plural: Lo Social son los procesos por los que se realizan los seres vivos CON, EN, POR, PARA, otros seres vivos." (Durand, 2001. p. 13)

Según la teoría de lo Social la condición necesaria para la existencia de lo social, es que el ser vivo es deficiente para ser por sí mismo y requiere de otro u otros para subsistir o ser. En la investigación los trabajadores mineros son deficientes para realizar todo el proceso minero, lo mismo la empresa también es deficiente para impulsar sus objetivos económicos sin los trabajadores, por tanto, es necesaria la complementación entre la empresa y los trabajadores. En este proceso es la empresa como organizadores e integradores, los encargados de dar dirección a los procesos, los responsables del bienestar psicosocial de los trabajadores, que debe traducirse en la psicología de los trabajadores.

La relación entre la Gestión empresarial y los trabajadores mineros, para ser eficaz, eficiente, la gestión empresarial debe encargarse de establecer las condiciones para que el trabajo de los mineros se cumpla con los requisitos necesarios para garantizar su salud, seguridad, capacitación, economía, y para cumplirlos dar los medios y condiciones para hacer realidad, así, sentar las bases 
sociales de su bienestar psicosocial. La gestión de la empresa debe tener en cuenta que los trabajadores mineros, por las características de su trabajo, más en zonas subterráneas, o en socavón, no sólo están expuestos a riesgos físicos, sino, también a riesgos de carácter psicológico, este aspecto, puede darse incluso a pesar que la gestión de la empresa cumpla con los requisitos de la seguridad física de los trabajadores. Así, el artículo expone los resultados de la investigación sobre el bienestar psicosocial de trabajadores mineros, teniendo presente su desarrollo y autorrealización personal, al estar expuestos a sufrir frustraciones psicosociales por la naturaleza de su trabajo.

\section{La teoría de la gestión basada en la calidad}

El artículo, Desarrollo y Autorrealización Personal de los Trabajadores Mineros de Socavón en una Empresa Minera con una Gestión de Calidad, pone énfasis en la visión y método de una gestión de Calidad, porque es el liderazgo de la Alta dirección ,la responsable de prevenir la seguridad y por tanto el bienestar psicosocial de los trabajadores en una empresa. Se considera que la gestión de la calidad, es un sistema de dirección que implanta la calidad en toda la empresa, como medio para conseguir los objetivos de calidad, caminando hacia la mejora continua en todos los niveles organizativos y utilizando todos los recursos disponibles con el menor coste posible.

De los catorce puntos desarrollados por (Deming 1986), se hace énfasis, en función de la investigación, los siguientes: 1. Crear Constancia de propósito para la mejora de productos y servicios. Sugiere que más que dinero una empresa mantenerse en el negocio y brindar empleo por medio de la innovación, investigación, la mejora constante y el mantenimiento. 5. Mejorar constantemente y por siempre el sistema de producción y servicios. La mejora no es un esfuerzo de una vez, 6. Instituir la capacitación. El trabajador debe ser capacitado para saber cómo hacer su labor. 7. Instituir el Liderazgo. Dirigir consiste no en la mera supervisar y castigo, sino en ayudar al personal a mejorar su trabajo. 8. Eliminar el temor. Para garantizar calidad y más productividad, es necesario que la gente se sienta segura.12. Remover las barreras que impiden el orgullo de un trabajo bien hecho. La gente desea hacer un buen trabajo y le mortifica no hacerlo. 13. Instituir un programa riguroso de educación y recapacitación.

\section{Objetivos}

Del conjunto de Objetivos planteados en la investigación, el presente artículo determina dos objetivos, relativos al desarrollo y autorrealización de los mineros:

1. Conocer el grado de dirección de vida, de metas trazadas, de los trabajadores mineros de socavón de Cerro Lindo de la compañía minero MILPO, S.A.A. en el curso del año 2015. 
2. Establecer el grado de crecimiento personal y desarrollo de sus potencialidades de los trabajadores mineros de socavón de Cerro Lindo, de la compañía minera MILPO, S.A.A. en el año 2015.

\section{METODOLOGÍA}

\section{Caso: La unidad minera Cerro Lindo de la Compañía Minera Milpo S.A.A.}

El caso lo constituye los trabajadores mineros de la unidad minera Cerro Lindo de la Compañía Milpo S.A.A. comprendidos en la Política Corporativa, Código de Conducta y Reglamente Interno de Trabajo de la empresa. Sobre la mina Cerro Lindo la empresa, señala: "El 20 de julio de 2007, Cerro Lindo se convirtió en la primera mina del País que no utiliza agua de río en sus operaciones".

\section{Tabla 1}

Ficha Técnica dela Unidad Cerro Linda S.A.A.

\begin{tabular}{lll}
\hline $\mathrm{N}^{\circ}$ & Elementos de identificación & Descripción \\
\hline 1 & Ubicación & Distrito de Chavín, provincia de Chincha, departamento de Ica \\
2 & Altitud & 1,820 m.s.n.m. \\
3 & Inicio de operaciones & Julio de 2007 \\
4 & Características & Mina polimetálica subterránea. \\
4 & Producción & Plomo, zinc y cobre, con un método de subniveles a través de un \\
5 & Volumen de producción & sistema de fajas transportadoras \\
\hline
\end{tabular}

Fuente. Página oficial web de la Compañía Milpo S.A.A.: http://www.milpo.com.pe/Content/Index.aspx?aID=115

\section{Diseño de la investigación}

El Enfoque Metodológico de la investigación es el Cuantitativo Deductivo, acorde a este enfoque, los objetivos son concretados mediante el método estadístico. El Tipo de Investigación a realizarse es el No Experimental, acorde a este tipo la investigación se alcanza los objetivos dejará intacto a su objeto, La Clase de Investigación es transversal, se hace corte en el tiempo del objeto, en relación al año 2014-2015. El Alcance de la Investigación, acorde a los objetivos planteados es Descriptivo, La investigación describe la realidad social de bienestar psicosocial de los trabajadores mineros y mide el bienestar psicológico de los trabajadores. El método para obtener y procesar los datos o información, conforme el enfoque metodológico cuantitativo-deductivo, es el Estadístico Descriptivo. Conforme este método se establece una población de la investigación, con los respectivos criterios de inclusión y exclusión. (Hernández, Fernández, \& Baptista, 2003).

\section{Población de la investigación}

\section{Población en general}

La población en general lo constituyen los trabajadores de la Unidad Mina Cerro Lindo de la compañía minera MILPO S.A.A., ubicada en el distrito de Chavín, provincia de Chincha, Departamento de Ica, que entre trabajadores administrativos y trabajadores mineros de socavón, son un total de 250 trabajadores. 
Tabla 2

Población en general de la investigación.

\begin{tabular}{lll}
\hline $\mathrm{N}^{\circ}$ & Trabajadores de cerro lindo & Número de trabajadores \\
\hline 1. & Administrativos y Servicios & 99 \\
2. & Trabajadores mineros socavón & 151 \\
& Total & 250 \\
\hline
\end{tabular}

\section{Población objetivo}

La población objetivo de la investigación se determina estableciendo los respectivos criterios de exclusión e inclusión. Por los Criterios de exclusión de la población objetivo no se considera a los trabajadores administrativos y de servicios que no realizan labores de trabajo en socavón, quedan excluidos de la población objetivo a investigar. Por los Criterios de inclusión de la población objetivo. Se determina los trabajadores mineros de la mina Cerro Lindo, dedicados a la extracción de minerales, en el socavón, en turnos diurnos y nocturnos, nombrados y contratados, constituyen la población objetivo de la presente investigación, siendo su número de 151 trabajadores mineros.

Tabla 3

Población objetivo.

\begin{tabular}{lll}
\hline $\mathrm{N}^{\circ}$ & Población objetivo & Número \\
\hline 1. & Trabajadores mineros de socavón & 151 \\
& Total & 151 \\
\hline
\end{tabular}

\section{Unidad de análisis de la Población Objetivo}

La unidad de análisis de la investigación lo constituye cada trabajador minero de la mina Cerro Lindo que cumpla el requisito de inclusión. Se considera que cada trabajador contiene la información requerida.

\section{Muestra de la población objetivo}

Por el número de la población objetivo, 151 trabajadores mineros, no requiere de una muestra aleatoria representativa, y se abarca al $100 \%$ de la población, por lo que el método estadístico es el descriptivo y no el probabilístico.

\section{Instrumento}

La investigación utiliza para recolectar la información de un Instrumento consistente en un Cuestionario de preguntas de estructura cerrada, que se aplicó como encuestas directas a la población objetivo. Para la adopción se ha tenido cuenta el siguiente cuadro, deducido de la base teórica, donde se relacionan los aspectos sociales con los psicológicos, para dar como resultado la realidad psicosocial de los trabajadores de la investigación. 
Tabla 4

Relaciones entre lo social y sus efectos psicológicos.

\begin{tabular}{llll}
\hline $\mathrm{N}^{\circ}$ & Aspectos sociales & $\mathrm{N}^{\circ}$ & Aspectos psicológicos \\
\hline 1 & Económicos Laborales & 1 & Satisfacción-malestar \\
2 & Salud & 2 & Satisfacción-malestar \\
3 & Beneficios Sociales & 3 & Con beneficios - Sin beneficios \\
4 & Educación & 4 & Con perspectivas - Sin \\
5 & Recreación & 5 & Con medios - sin medios \\
6 & Vivienda y servicios & 6 & Adecuados - no adecuados \\
\hline
\end{tabular}

\section{El modelo multidimensional psicológico - Instrumento Ryff}

La investigación tuvo en cuenta que la gestión moderna y de calidad de la Compañía, MILPO S.A.A, brinda los servicios sociales a sus trabajadores, sin embargo, por las características del trabajo minero de riesgo, especialmente en los trabajos en socavón, se aplica el instrumento Riff para conocer el estado de bienestar psicosocial de los trabajadores mineros objetivo de la investigación. El instrumento consta de 6 dimensiones, 1) auto-aceptación, 2) relaciones positivas con otras personas, 3) autonomía, 4) dominio del entorno, 5) propósito de vida 6) y crecimiento personal. De estas dimensiones el presente artículo se concentra en las dos últimas. Propósito de vida. O tener metas y sensación de una dirección de la vida, asociado a la motivación para actuar y desarrollarse. Las personas necesitan marcarse metas, definir una serie de objetivos que les permitan dotar a su vida de un cierto sentido. Crecimiento personal. Asociado a la idea de evolución y aprendizaje positivo de la persona. El funcionamiento positivo óptimo no sólo requiere de las características señaladas, también el empeño por desarrollar sus potencialidades, por seguir creciendo como persona y llevar al máximo sus capacidades.

\section{El instrumento, escalas de bienestar psicológico de RYFF}

El instrumento Ryff es un Cuestionario de 39 preguntas valorativas, relativas a examinar la psicología en la perspectiva del bienestar psicológico. Cada pregunta valorativa conlleva a 6 alternativas de escala, para ser marcadas con una x en el grado de la escala, por el encuestado. Los grados de escala son: 1) Totalmente en desacuerdo, 2) Desacuerdo, 3) En parte en desacuerdo, 4) En parte de acuerdo, 5 De acuerdo, 6) Totalmente de acuerdo. Las dimensiones de graduación de las dimensiones determinadas son: Crecimiento personal. Por encima de $37-28$ puntos tiene muy buen crecimiento personal, por debajo de $27-28$ puntos tiene poco crecimiento personal. Propósito de vida. Por encima de 32 puntos tiene mucho sentido su vida y tiene metas. Por debajo de 22 puntos tiene poco sentido su vida, no tiene metas y propósitos vitales definidos. 


\section{Validación del instrumento}

La validación del instrumento lo asume el artículo a partir de la investigación realizada, en términos de criterio, constructo y contenido. Los dos primeros criterios se dan en base a la matriz de consistencia y al marco teórico, que validan el instrumento por estar conforme a lo metodológico (validez de constructo) y a lo teórico (validez de criterio). La tercera validación del instrumento correspondiente al contenido se ha aplicado la encuesta a un piloto de 10 trabajadores, que reúnan los criterios de inclusión, con está validación se aplica el instrumento a toda la población objetivo.

El instrumento demostró ser confiable y válido, siguiendo la fórmula básica, $\mathrm{X}=\mathrm{t}+\mathrm{e}$, se obtuvo $10=9+1$ (Donde $\mathbf{X}$ es los valores observados, $\mathbf{t}$ los valores verdaderos y e el grado de error). Se halló un coeficiente de confiabilidad del $90 \%$ lo que nos da un nivel aceptable (Sampieri, 2010).

\section{Trabajo de campo}

Validado el instrumento, se procedió a su aplicación, mediante la técnica de las encuestas - entrevistas a las unidades de análisis de la población, que se caracterizó por ser directa, en el trabajo de campo, en el centro minero. Así, se da una relación directa entre el encuestador con cada unidad de análisis, evitando posibles sesgos dados por la aplicación indirecta. Realizado el trabajo de campo que duro 1 año abril 2015 a marzo 2016, el horario que se aplicó el instrumento de 6:00 a.m. A 6.30, 6:00 pm a 7:00 p.m. durante 7 meses. Se pasa al procesamiento de la información recolectada.

\section{Procesamiento de los datos recolectados}

La información recolectada en el trabajo de campo se procesará mediante el programa estadístico SPSS, sobre cuyos resultados se procederá a presentar los resultados, su análisis, interpretación y discusión.

\section{RESULTADOS}

En el Figura 1 se observa que, el $79.42 \%$ de trabajadores de 1 a 4 meses de tiempo de servicio no tienen metas claras y menos objetivos para sus vidas. Un $74.08 \%$, de 1 a 2 años de tiempo de servicio, de la misma manera, no dan sentido a su vida. Un $73.75 \%$, de 2 años a tres años de tiempo de servicio de trabajo, se sienten totalmente vacíos por dentro, no saben lo que quieren, y un $69.39 \%$, de 1 a 5 meses de tiempo de servicios, sienten que su vida no es satisfactoria. El grado de dirección de vida, de metas trazadas en los trabajadores mineros de socavón de Cerro Lindo, es de Bajo nivel por no tener un propósito de vida. El resultado expuesto concreta el objetivo 1 del artículo. En efecto los trabajadores no tienen metas y sensación de una dirección en sus vidas, asociado a la motivación para actuar y desarrollarse. 


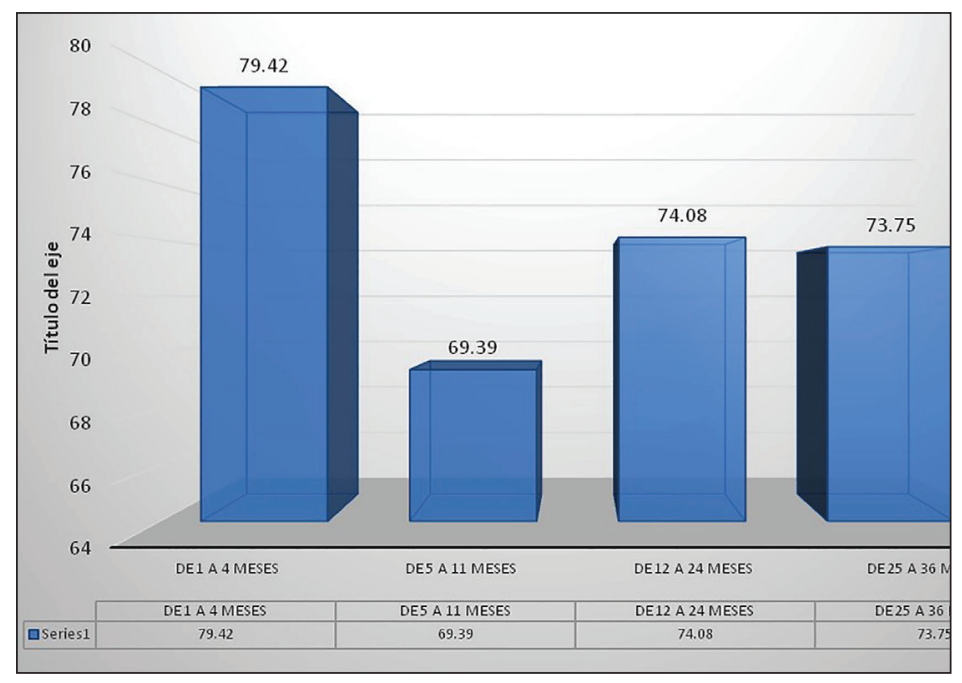

Figura 1. Propósito de vida - tiempo de servicio.

En el Figura 2 se observa, un 73.17, los trabajadores entre, 20 y 29 años de edad, no tienen la predisposición de crecer como ser humano, y, de la misma manera, un $64,67 \%$, entre las edades de 30 a 34 años, tienen poco o nada el interés de desarrollar sus potencialidades.

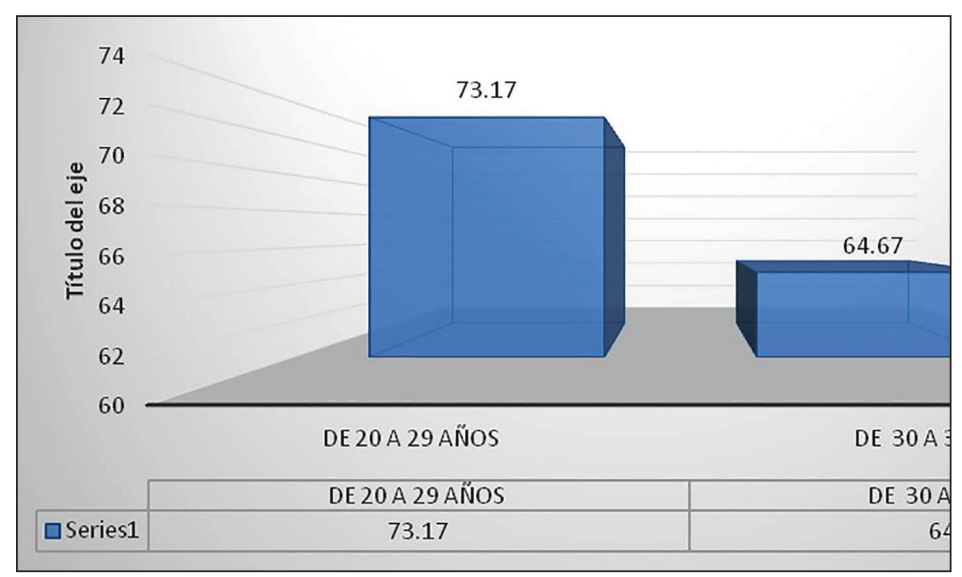

Figura 2. Crecimiento personal - edad.

En el Figura 3 se puede observar, que un $80.92 \%$, con 25 a 36 meses de tiempo de trabajo, demuestran que no tienen el deseo de desarrollar sus potencialidades. Un 79.86, de la misma manera, no tienen interés en su desarrollo personal. Un $72.33 \%$, no les interesa poner de su parte para salir adelante, y un $63.93 \%$, no muestran interés alguno en su persona. 


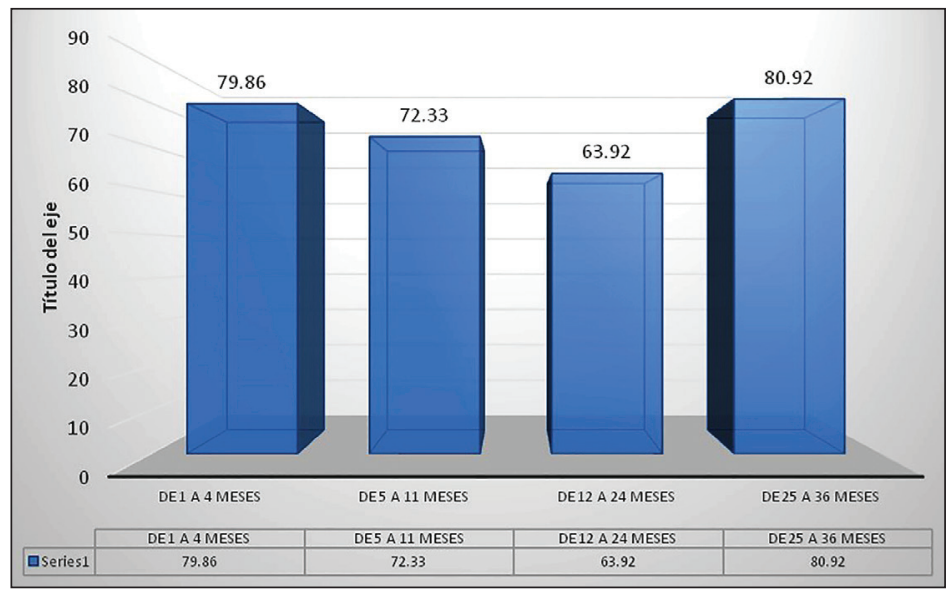

Figura 3. Crecimiento personal - tiempo de servicio.

En el Figura 4 se observa, que un $78.49 \%$, no les interesa generar condiciones para su crecimiento personal, les aburre las capacitaciones, manifiestan, que de nada les sirve porque no por eso van a ganar más. Un $77.13 \%$, con estudios técnicos, creen que es bueno buscar el desarrollo humano, pero, no tienen tiempo para eso, ni menos quisieran cambiar su forma de pensar. Un $77.08 \%$, con estudios superiores incompletos, manifiestan, que, así como' son, están bien, y, que nadie tiene que ver con su persona es su vida. El 57.81\%, de trabajadores con estudios superior, manifiestan que más adelante puede ser, pero, ahora no tienen interés en saber de cambios en su persona.

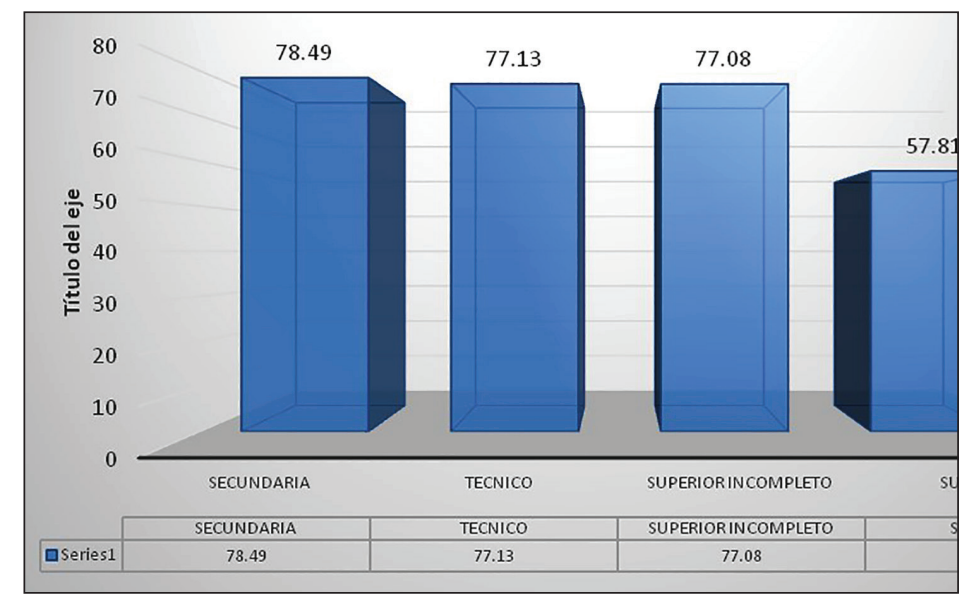

Figura 4. Crecimiento personal - educación. 
Con los resultados expuestos se concreta el objetivo 2. En efecto, los resultados comprueban lo formulado, en el sentido que no se da el crecimiento personal, para la evolución y aprendizaje positivo de la persona.

\section{DISCUSIÓN}

La aplicación del instrumento de Riff pone al descubierto que altos porcentajes de los trabajadores no se plantearon objetivos de metas no tienen propósito de vida, son personas que viven en constante quejas, y no se arriesgan a enfrentar los retos de su desarrollo.

El artículo, en base a los resultados de la investigación, "Bienestar Psicosocial de los Trabajadores del Centro Minero Milpo-Chincha alta-2015”, en las dimensiones de Propósito de Vida, y Crecimiento Personal, correspondientes al desarrollo y autorrealización, descubre una realidad social problemática, preocupante. Los cuadros estadísticos expuestos nos muestran las tendencias dominantes negativas, en relación al desarrollo y la autorrealización personal de los trabajadores mineros de socavón, en una empresa minera que manifiesta tener una gestión de Calidad.

El otro aspecto relativo a las funciones de una empresa minera moderna que implementa una gestión de Calidad, al no lograr captar este aspecto psicológico y social de sus trabajadores, a su vez, no sólo pone en riesgo la seguridad de sus trabajadores sino de toda la empresa. La empresa con una gestión de Calidad, cumpliendo los 14 Puntos, especialmente los Puntos: 1, 5, 6, 7 y el 13, podría velar por el bienestar psicosocial de los trabajadores, con una política destinada al desarrollo y autodesarrollo personal, para lograr una eficaz complementación entre la empresa y sus trabajadores.

Los resultados expuestos muestran una contradicción, o un profundo desajuste, entre el bienestar psicológico de los trabajadores y el bienestar psicosocial, de tal modo que al no darse el primero tampoco se da el segundo, a pesar, de los servicios y condiciones sociales que brinda la empresa, conllevando a plantearse está problemática desde una perspectiva diferente.

\section{CONCLUSIONES}

Las siguientes son las conclusiones alcanzadas por la investigación en relación a los objetivos propuestos:

- El propósito de vida del trabajador minero de socavón en la mina Cerro Lindo, acorde al instrumento utilizado de Ryff, determina que el bienestar psicosocial que responde al propósito de vida es deficitario.

- En conjunto, respecto al bienestar psicológico, acorde al instrumento utilizado de Ryff, se debe concluir, que es deficitario. 
- La conclusión expuesta a partir de los resultados, conlleva al planteamiento que no son suficientes los servicios sociales, la normatividad legal, para garantizar el bienestar psicológico de los trabajadores, que es necesario ahondar más en su problemática, para poder establecer una relación directa entre bienestar psicológico y bienestar psicosocial. En el caso de la investigación, el bienestar psicológico deficitario genera un bienestar también deficitario en el ámbito psicosocial.

\section{RECOMENDACIONES}

En base a los resultados, es necesario hacer las siguientes recomendaciones:

1. En relación a la Gestión Empresarial, se debe impulsar investigaciones y estudios sobre la problemática social y psicológica de los trabajadores mineros, para lograr la superación de las tendencias psicológicas y psicosociales negativas. Es necesario revertir esta realidad psicosocial, mejorando la cultura organizacional, para poder lograr complementaciones exitosas, generando beneficios tanto para la empresa y para los trabajadores.

2. En relación a los trabajadores, existiendo una tendencia general de déficit en su bienestar psicosocial, siendo especial en relación al crecimiento personal, debe atenderse con prioridad en todas áreas, con talleres especiales y con atención terapéutica personal.

3. Por último, siendo esencial el propósito de vida, se debe impulsar actividades de confraternidad, conversatorios, que tengan la finalidad de hacer reflexionar y evaluar los objetivos personales de los trabajadores.

\section{REFERENCIAS}

García-Viniegras, C. y González-Benítez, I. (2000). La Categoría Bienestar Psicológico. Revista Cubana de Medicina General Integral, 16 (6). pp. 586-592. La Habana.

Deming, E. (1986). Calidad, Productividad y Competitividad. España: Ediciones Díaz de Santos, S.A.

Durand, J. (2001). El Misterio de lo Social - Teoría de lo Social. Perú: Fondo Editorial Paradigmas.

Hernández, R.; Fernández, C.; y Baptista, P. (2003). Metodología de la Investigación. México: Mc Graw Hill.

Jaramillo, V. A. (2005). Factores de Riesgo Psicosocial. Bogotá: Publicaciones Gabo.

Kayes, R. (1995). Bienestar Psicológico con la Personalidad. Chicago: USA.

Pacheco, P. O. (2015). El papel de las creencias de seguridad en los resultados de seguridad influyentes en el sector minero en los países de América del sur. Guayaquil: Ecuador.

Sampieri, R, H (2010). Metodología de la investigación. México: Mc Graw Hill. 